\title{
Clinically Delivered Treatment for Glioma Patients on a Hybrid Magnetic Resonance Imaging (MRI)- Linear Accelerator (MR-Linac) and a Cone Beam CT (CBCT)-Guided Linac: Dosimetric Comparisons with In Vivo Skin Dose Correlation
}

Michael H. Wang

Sunnybrook Health Sciences Centre

Anthony Kim

Sunnybrook Health Sciences Centre

Mark Ruschin

Sunnybrook Health Sciences Centre

Hendrick Tan

Sunnybrook Health Sciences Centre

Hany Soliman

Sunnybrook Health Sciences Centre

Sten Myrehaug

Sunnybrook Health Sciences Centre Jay Detsky

Sunnybrook Health Sciences Centre

Zain Husain

Sunnybrook Health Sciences Centre

Eshetu G. Atenafu

University Health Network

Brian Keller

Sunnybrook Health Sciences Centre

Arjun Sahgal

Sunnybrook Health Sciences Centre

Chia-Lin Tseng ( $\nabla$ chia-lin.tseng@sunnybrook.ca )

Sunnybrook Health Sciences Centre https://orcid.org/0000-0002-3700-0886

Research Article 
Keywords: MRI-guided radiotherapy, MR-Linac, Glioma, Interface effects, Electron return effect, Monte Carlo

Posted Date: August 17th, 2021

DOl: https://doi.org/10.21203/rs.3.rs-807483/v1

License: (c) (1) This work is licensed under a Creative Commons Attribution 4.0 International License. Read Full License 


\section{Abstract \\ Background}

Magnetic Resonance Imaging (MRI)-Linear Accelerator (MR-Linac) radiotherapy requires special consideration for secondary electron interactions within the magnetic field, which can alter dose deposition at air-tissue interfaces.

\section{Methods}

Thirty-seven consecutive glioma patients treated during their radiotherapy course with at least one fraction delivered on MR-Linac or Cone Beam CT (CBCT)-guided Linac, were analyzed. Treatment planning for both systems were completed prior to radiotherapy initiation and approved for clinical delivery using commercial treatment planning systems (TPS): a Monte Carlo calculation-based or convolution calculation-based TPS for MR-Linac or CBCT-Linac, respectively. Dosimetric parameters for planning target volume (PTV), organs-at-risk (OARs), and air-tissue interface were compared. In vivo skin dose during a single fraction of MR-Linac and CBCT-Linac treatment was measured using an Optically Stimulated Luminescent Dosimeter (OSLD) and correlated with TPS skin dose.

\section{Results}

Monte Carlo-based MR-Linac plans and convolution-based CBCT-Linac plans exhibited minimal differences in PTV and OAR parameters. However, MR-Linac plans had greater doses within tissues surrounding air cavities $(1.52$ Gy higher mean Dmean, $p<0.0001)$ and skin $(1.10$ Gy higher mean Dmean, $p<0.0001)$. In vivo OSLD skin readings were $14.5 \%$ greater for MR-Linac treatments $(p=0.0027)$, and were more accurately predicted by Monte Carlo-based calculation $(\rho=0.95, p<0.0001)$ vs. convolutionbased $(\rho=0.80, p=0.0096)$.

\section{Conclusions}

The magnetic field's dosimetric impact was minimal for PTV and OARs in glioma as compared to standard CBCT-Linac treatment plans. However, skin doses were significantly greater with the MR-Linac and correlated with in vivo measurements. Future MR-Linac planning processes are being designed to account for skin dosimetry and treatment delivery.

\section{Introduction}

Modern radiotherapy with selective incorporation of Magnetic Resonance Imaging (MRI) during the treatment course has shown promise for evaluating glioma tumor dynamics [1], predicting glioma Loading [MathJax]/jax/output/CommonHTML/jax.js is for dose escalation based on functional imaging. Typically, 
no more than 2 to $3 \mathrm{MR}$ images were obtained during a 6-week radiation treatment course due to practicalities and cost of obtaining repeat MRIs. With the advent of integrated MRI-linear accelerator (MRLinac) delivery systems, this technology has the potential for the first time to allow daily acquisition of high field strength (1.5 Tesla) diagnostic quality MRI to enable adaptive radiotherapy, to individualize the radiation treatment plan and to incorporate functional imaging. At our institution, we have been developing the application of the Unity MR-Linac (Elekta AB, Stockholm, Sweden) to brain tumors, and have treated $\sim 150$ glioma patients with either 3 or 6 weeks of daily fractionated standard radiotherapy with concurrent temozolomide.

The challenge of delivering radiation within a high MRI field strength environment is the influence of the Lorentz force on dose deposition. The Electron Return Effect (ERE) refers to secondary electrons exiting tissue into air being curved back to deposit dose at the tissue surface due to the Lorentz Effect, which results from the presence of the strong magnetic field placed perpendicularly to the beam direction [3$11]$.

Until now, the ERE has been investigated in phantom studies [12], as well as in simulated dosimetric studies [13-17] with research versions of MR-Linac planning software prior to the release of the current clinical treatment planning version. The purpose of the current study is to report on those glioma patients treated on the MOMENTUM prospective registry study (Clinicaltrials.gov: NCT04075305) [18] who received at least one fraction of radiotherapy on both the MR-Linac and a Cone Beam CT (CBCT)-guided Linac: 1) the dosimetric impact of the magnetic field on the target, organs-at-risk (OARs), skin and tissues surrounding air cavities, and; 2) to compare skin dose modelled from the treatment planning software with in vivo skin dose measurements.

\section{Materials And Methods}

\section{Patient Population}

This consecutive case series of glioma patients treated between July 2019 and February 2021 consented to the institutional research ethics board approved prospective MOMENTUM trial. Patients were treated on the Unity MR-Linac (Elekta AB, Stockholm, Sweden) and our practice was to create a clinical backup conventional CBCT-Linac plan during treatment planning. On days when the MR-Linac was scheduled for maintenance or had a service issue such that treatment would otherwise be delayed, patients were irradiated on an Elekta CBCT-Linac (Elekta AB, Stockholm, Sweden) for that particular radiotherapy fraction. Thirty-seven glioma patients treated with at least one fraction on both the MR-Linac and CBCTLinac for their adjuvant radiotherapy at our institution met these criteria. All patients completed maximal safe surgical resection followed by either 3 or 6 weeks of adjuvant radiotherapy according to standard practices [19-20].

\section{Radiotherapy Simulation and Target Definition}


All patients were simulated with a treatment planning CT (Brilliance, Philips Healthcare, Best, Netherlands) with a slice thickness of $1.0 \mathrm{~mm}$, and immobilized with a thermoplastic head immobilization device (CIVCO Medical Solutions, Kalona, lowa, USA). MRI simulation at 1.5 Tesla (Ingenia, Philips Healthcare, Best, Netherlands) was also performed in the treatment position with the immobilization device applied. Standard volumetric axial T1 gadolinium-enhanced and T2 FLAIR sequences with a slice thickness of $1.0 \mathrm{~mm}$ were acquired. CT images and MRI sequences were registered and fused based on rigid mutual information registration within a region of interest box defined around the tumor [21].

Delineation of OARs, gross tumor volume (GTV), and clinical target volume (CTV) were defined based on glioma consensus contouring guidelines [22]. Planning target volume (PTV) was generated as a 0.4-cm isotropic expansion of the CTV.

\section{Radiotherapy Planning}

Treatment planning for the MR-Linac was completed on the Monaco treatment planning system (TPS) (Monaco v5.40, Elekta AB, Stockholm, Sweden). The Monte Carlo algorithm [23] accounts for magnetic field effects for the MR-Linac. A TPS based on a convolution-superposition dose calculation algorithm (Pinnacle v9.8, Philips Healthcare, Eindhoven, Netherlands) was used for planning on the CBCT-Linac (Fig. 1). All MR-Linac plans used $\geq 9$ coplanar non-opposing Intensity-Modulated Radiation Therapy (IMRT) beams, and were optimized to achieve at least $90 \%$ of the prescription dose covering $99 \%$ of PTV (D99\% $>90 \%)$, less than $5 \%$ of PTV getting $105 \%$ of the prescription dose (V105\% $<5 \%)$, and less than $110 \%$ of the prescription dose to $0.1 \mathrm{cc}$ volume of PTV (D0.1cc $<110 \%$ ), as outlined by the MR-Linac consortium clinical treatment planning document. All CBCT-Linac plans used $\geq 7$ coplanar non-opposing IMRT beams, and were optimized to achieve at least $95 \%$ of PTV covered by $95 \%$ of the prescription dose (V95\% $\geq 95 \%$ ), and at most $1 \%$ of PTV getting $105 \%$ of the prescription dose (V105\% $\leq 1 \%)$. Dose to OARs were constrained according to our institutional protocols (Supplementary Material, Table 1). 
Table 1

Patient Characteristics

\begin{tabular}{|ll|}
\hline Characteristic & Total (N= 37) \\
\hline Age & $22(59.5 \%)$ \\
$60-69$ years & $7(18.9 \%)$ \\
$\geq 70$ years & $8(21.6 \%)$ \\
\hline Gender & \\
Male & $25(67.6 \%)$ \\
Female & $12(32.4 \%)$ \\
WHO Grade & \\
II & $9(24.3 \%)$ \\
III & $4(10.8 \%)$ \\
IV & $24(64.9 \%)$ \\
\hline Prescription & $8(21.6 \%)$ \\
40 Gy in 15 fractions & $15(40.5 \%)$ \\
54 Gy in 30 fractions & $13(35.1 \%)$ \\
60 Gy in 30 fractions & $1(2.7 \%)$ \\
59.4 Gy in 33 fractions & \\
\hline
\end{tabular}

\section{Radiotherapy Plan Evaluation}

MR-Linac Monaco plans and CBCT-Linac Pinnacle plans for each patient were evaluated using cumulative dose-volume histogram (DVH) (Fig. 2) parameters chosen a priori. For targets, dosimetric parameters of interest were extracted and compared from reference plans for the PTV, CTV and GTV, including: 1) Volumes of tissue receiving at least $100 \%, 95 \%, 50 \%$ of the prescription dose $(\mathrm{V} 100 \%, \mathrm{~V} 95 \%$, V50\%); and 2) Minimum doses to $2 \%, 5 \%, 50 \%, 95 \%, 98 \%$ of the target volume (D2\%, D5\%, D50\%, D95\%, D98\%). Dose homogeneity was assessed using Homogeneity Index ( $\mathrm{Hl}$; Supplementary Material, Eq. 1), with lower HI values (closer to 1.0) indicating more homogeneous dose distribution. Dose falloff outside of the target was assessed using Gradient Index (Gl; Supplementary Material, Eq. 2), with lower GI values characterizing a sharper dose falloff outside of the target. Dose conformity was assessed using Conformity Index (Cl; Supplementary Material, Eq. 3), with $\mathrm{Cl}$ values closer to 1.0 signifying better conformity. OARs for each patients' clinical treatment plans were extracted and compared including: Maximum dose to $0.03 \mathrm{cc}$ volume of Lens, Globe, Optic chiasm, Optic nerve (Lens D0.03cc, Globe

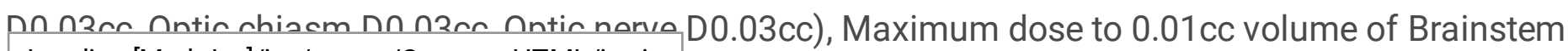
Loading [MathJax]/jax/output/CommonHTML/jax.js 
(Brainstem D0.01cc), and Mean dose to each cochlea (Cochlea Dmean). The maximum dose to a small but finite volume of an OAR was defined so as to partially manage the statistical noise inherent in a Monte Carlo TPS.

\section{Skin Dosimetry Assessment}

Skin volumes were generated as a $5 \mathrm{~mm}$ rim of tissue contracted from the patient body surface (Supplementary Material, Fig. 1). Tissues around air cavities were generated as a $5 \mathrm{~mm}$ rim of tissue expanded from air cavity volumes (e.g. nasopharynx, paranasal sinuses). Dosimetric parameters analyzed included: Mean dose and Maximum dose to 2cc volumes of tissues surrounding air cavities and skin contours (Air cavity Dmean, Air cavity D2cc, Skin Dmean, Skin D2cc), as well as the volume of skin receiving 20 Gy (Skin V20Gy).

For 10 randomly selected patients, in vivo skin dose measurements during one fraction of MR-Linac treatment and one fraction of CBCT-Linac treatment were obtained using Optically Stimulated Luminescent Dosimeters (OSLDs; nanoDots, Landauer, Glenwood, IL, USA) placed in a defined location on the patient's skin near the PTV (Supplementary Material, Fig. 2). OSLD dose point in the TPS was defined as the placement location on the treatment unit, at $0.6 \mathrm{~mm}$ depth from the patient surface. In the TPS, the patient surface was defined as the $0.6 \mathrm{~g} / \mathrm{cc}$ boundary between tissue and air. The $0.6 \mathrm{~mm}$ depth is the water equivalent depth of an OSLD taking into account its plastic casing, and the accuracy was rated to be $\pm 3 \%$ [12]. Dose delivered to skin, as measured by the OSLD, were correlated to both corresponding clinical treatment plans to determine which TPS best predicted the in vivo dose measurement.

\section{Statistical Analysis}

Descriptive statistics were used to summarize the dosimetric parameters. Assumption of normality was assessed using the Smirnov-Kolmogorov test. Student's t-paired test or Wilcoxon signed-rank test, as appropriate based on normality assumption, were used to compare dosimetric parameters between MRLinac plans and CBCT-Linac plans. Spearman's correlation was used to assess the relationship between in vivo OSLD measurements and TPS skin dose. All statistical tests were two-sided, and threshold used for statistical significance was $p<0.05$. Statistical analyses were performed using version 9.4 of the SAS system for Windows (2002-2012 SAS Institute, Inc., Cary, NC, USA).

\section{Results}

\section{Target Volumes and Organs at Risk}

Thirty-seven glioma patients treated with at least one fraction on both the MR-Linac and CBCT-Linac were analyzed (Table 1). Comparisons of PTV dosimetric parameters between MR-Linac and CBCT-Linac are detailed in Table 2a. There is no statistical difference in PTV V100\%, V95\%, V50\%, D98\%, and D95\% ( $>$ > 0.05). Small but statistically significant differences were observed in mean PTV D50\% $(p<0.0001)$, D $5 \%$ Loading [MathJax]/jax/output/CommonHTML/jax.js R-Linac plans compared with CBCT-Linac. MR-Linac dose 
distributions had higher $\mathrm{HI}(p=0.0007)$, better $\mathrm{Cl}(p=0.0064)$, and equivalent $\mathrm{GI}(p>0.05)$ compared to CBCT-Linac.

Table 2

a. Dosimetric impact of magnetic field on target volume parameters in treated glioma patients

\begin{tabular}{|lllll|}
\hline PTV & $\begin{array}{l}\text { MR-Linac } \\
(\text { Mean } \pm \text { ME) }\end{array}$ & $\begin{array}{l}\text { Conventional Linac } \\
(\text { Mean } \pm \text { ME) }\end{array}$ & $\begin{array}{l}\text { Paired Difference } \\
(\text { Mean } \pm \text { ME) }\end{array}$ & P value \\
\hline V100\% (cc) & $265.94 \pm 41.15$ & $265.28 \pm 41.43$ & $0.66 \pm 3.87$ & 0.9705 \\
\hline V95\% (cc) & $278.48 \pm 42.19$ & $269.44 \pm 43.00$ & $9.04 \pm 14.54$ & 0.2920 \\
\hline V50\% (cc) & $280.07 \pm 42.19$ & $279.96 \pm 42.17$ & $0.11 \pm 0.18$ & 0.5000 \\
\hline D98\% (Gy) & $52.19 \pm 2.46$ & $51.57 \pm 2.43$ & $0.62 \pm 0.66$ & 0.5786 \\
\hline D95\% (Gy) & $53.11 \pm 2.50$ & $52.67 \pm 2.47$ & $0.44 \pm 0.46$ & 0.1090 \\
\hline D50\% (Gy) & $54.67 \pm 2.57$ & $54.39 \pm 2.57$ & $0.27 \pm 0.10$ & $<0.0001$ \\
\hline D5\% (Gy) & $55.92 \pm 2.63$ & $55.17 \pm 2.60$ & $0.75 \pm 0.12$ & $<0.0001$ \\
\hline D2\% (Gy) & $56.28 \pm 2.65$ & $55.42 \pm 2.61$ & $0.86 \pm 0.12$ & $<0.0001$ \\
\hline Homogeneity Index & $1.05 \pm 0.01$ & $1.05 \pm 0.02$ & $0.01 \pm 0.01$ & 0.0007 \\
\hline Gradient Index & $1.07 \pm 0.04$ & $1.22 \pm 0.33$ & $-0.14 \pm 0.29$ & 0.8303 \\
\hline Conformity Index & $0.87 \pm 0.02$ & $0.83 \pm 0.03$ & $0.04 \pm 0.03$ & 0.0064 \\
\hline ME = Margin of error for $95 \% \mathrm{Cl}$ & & & \\
\hline Paired difference = MR-Linac - Conventional Linac & & \\
\hline
\end{tabular}


Table 2

b. Dosimetric impact of magnetic field on organs-at-risk (OARs) in treated glioma patients

\begin{tabular}{|lllll|}
\hline OARs & $\begin{array}{l}\text { MR-Linac } \\
\text { (Mean } \pm \\
\text { ME) }\end{array}$ & $\begin{array}{l}\text { Conventional } \\
\text { Linac } \\
\text { (Mean } \pm \text { ME) }\end{array}$ & $\begin{array}{l}\text { Paired } \\
\text { Difference } \\
\text { (Mean } \pm \text { ME) }\end{array}$ & P value \\
\hline Brainstem D0.1cc (Gy) & $47.27 \pm 4.48$ & $46.72 \pm 4.58$ & $0.55 \pm 0.79$ & 0.0307 \\
\hline Optic Chiasm D0.03cc (Gy) & $43.88 \pm 4.78$ & $42.78 \pm 5.24$ & $1.11 \pm 1.17$ & 0.5635 \\
\hline $\begin{array}{l}\text { Optic Nerve Right D0.03cc } \\
\text { (Gy) }\end{array}$ & $35.47 \pm 5.64$ & $35.36 \pm 6.09$ & $0.11 \pm 1.38$ & 0.1804 \\
\hline Optic Nerve Left D0.03cc (Gy) & $34.29 \pm 5.87$ & $34.64 \pm 6.14$ & $-0.35 \pm 1.26$ & 0.4858 \\
\hline Globe Right D0.03cc (Gy) & $24.90 \pm 4.51$ & $21.87 \pm 4.61$ & $3.03 \pm 1.69$ & 0.0009 \\
\hline Globe Left D0.03cc (Gy) & $22.26 \pm 3.54$ & $18.56 \pm 3.48$ & $3.70 \pm 1.98$ & 0.0005 \\
\hline Cochlea Right Dmean (Gy) & $18.25 \pm 6.70$ & $18.56 \pm 6.53$ & $-0.30 \pm 1.78$ & 0.5597 \\
\hline Cochlea Left Dmean (Gy) & $15.52 \pm 6.94$ & $16.39 \pm 7.05$ & $-0.87 \pm 1.80$ & 0.5046 \\
\hline Lens Right D0.03cc (Gy) & $7.15 \pm 0.79$ & $5.25 \pm 0.79$ & $1.89 \pm 0.67$ & $<0.0001$ \\
\hline Lens Left D0.03cc (Gy) & $7.43 \pm 0.75$ & $5.29 \pm 0.85$ & $2.14 \pm 0.71$ & $<0.0001$ \\
\hline ME = Margin of error for 95\% Cl & & & & \\
\hline Paired difference = MR-Linac - Conventional Linac & & & \\
\hline
\end{tabular}

Comparison of OAR dosimetric parameters between MR-Linac and CBCT-Linac are shown in Fig. 2a, and summarized in Table $2 \mathrm{~b}$. There was no statistical difference in the D0.03cc for the optic chiasm and optic nerves $(p>0.05)$, and no statistical difference in the mean dose to each cochlea $(p>0.05)$. Small but statistically significant differences were observed in clinically delivered D0.03cc for brainstem $(p=$ $0.0307)$, D 0.03cc for each globe ( $<<0.0010)$, and D0.03cc for each lens $(p<0.0001)$ for the MR-Linac.

\section{Tissues around Air Cavities and at Skin Surface}

The difference in dosimetric parameters for tissues surrounding air cavities and skin between MR-Linac and CBCT-Linac are shown in Fig. 2b, and summarized in Table 3a. For tissues surrounding air cavities, MR-Linac treatments had statistically significant higher Dmean $(p<0.0001)$ and D2cc $(p=0.0007)$ compared to CBCT-Linac. For skin tissues, MR-Linac treatments had statistically significant higher Dmean $(p<0.0001)$ and V20Gy $(p=0.0001)$ compared to CBCT-Linac, but no difference in D2cc $(p=$ 0.7975). 
Table 3

a. Dosimetric impact of magnetic field on tissues surrounding air cavities and at skin surface in treated glioma patients

\begin{tabular}{|lllll|}
\hline $\begin{array}{l}\text { Tissues Surrounding Air Cavities and at } \\
\text { Skin Surface }\end{array}$ & $\begin{array}{l}\text { MR-Linac } \\
\text { (Mean } \pm \\
\text { ME) }\end{array}$ & $\begin{array}{l}\text { Conventional } \\
\text { Linac } \\
\text { (Mean } \pm \text { ME) }\end{array}$ & $\begin{array}{l}\text { Paired } \\
\text { Difference } \\
\text { (Mean } \pm \text { ME) }\end{array}$ & $\begin{array}{l}\text { Palue } \\
\text { Air Cavity Dmean (Gy) }\end{array}$ \\
\hline $\begin{array}{l}12.05 \pm \\
2.27\end{array}$ & $10.53 \pm 2.08$ & $1.52 \pm 0.60$ & $<$ \\
\hline Air Cavity D2cc (Gy) & $\begin{array}{l}41.78 \pm \\
6.10\end{array}$ & $40.55 \pm 6.44$ & $1.23 \pm 0.98$ & 0.0007 \\
\hline Skin Dmean (Gy) & $\begin{array}{l}\text { 8.64 } \\
1.99\end{array}$ & $7.54 \pm 2.00$ & $1.10 \pm 0.54$ & $<$ \\
\hline Skin D2cc (Gy) & $\begin{array}{l}45.55 \pm \\
2.80\end{array}$ & $45.45 \pm 3.67$ & $0.11 \pm 0.85$ & 0.7975 \\
\hline Skin V20Gy (cm3) & $\begin{array}{l} \\
13.70\end{array}$ & $66.55 \pm 12.16$ & $19.04 \pm 11.45$ & 0.0001 \\
\hline ME = Margin of error for 95\% Cl & & & & \\
\hline Paired difference = MR-Linac - Conventional Linac & & & \\
\hline
\end{tabular}

Table 3

b. Comparison of predicted skin dose from TPS software and delivered skin dose measured from OSLD

\begin{tabular}{|lllll|}
\hline Skin Dose & $\begin{array}{l}\text { MR-Linac } \\
(\text { Mean } \pm \text { ME) }\end{array}$ & $\begin{array}{l}\text { Conventional Linac } \\
(\text { Mean } \pm \text { ME) }\end{array}$ & $\begin{array}{l}\text { Mean Difference } \\
\text { (Mean } \pm \text { ME) }\end{array}$ & $\begin{array}{l}\text { P } \\
\text { value }\end{array}$ \\
\hline TPS (Gy) & $35.0 \pm 7.7$ & $27.2 \pm 6.8$ & $7.8 \pm 4.1$ & 0.0020 \\
\hline OSLD (Gy) & $36.3 \pm 9.6$ & $31.7 \pm 8.8$ & $4.6 \pm 2.5$ & \\
\hline $\begin{array}{l}\text { Mean } \\
\text { Difference }\end{array}$ & $1.4 \pm 2.1$ & $4.0 \pm 5.2$ & \\
\hline P value & $\begin{array}{l}\text { Spearman } \rho=0.9500, \\
p<0.0001\end{array}$ & $\begin{array}{l}\text { Spearman } \rho=0.8000, p \\
<0.0096\end{array}$ & \\
\hline ME = Margin of error for $95 \% \mathrm{Cl}$ & & & \\
\hline
\end{tabular}

Differences between predicted skin dose from the TPS and delivered skin dose measured from OSLD are quantified in Table 3b. Mean skin dose as determined by the TPS was 7.8 Gy greater on MR-Linac plans $(95 \% \mathrm{Cl} 3.7-11.8 \mathrm{~Gy})$ compared with CBCT-Linac $(\mathrm{p}=0.0020)$. Mean in vivo OSLD skin readings were 4.6 Gy greater (14.5\% higher) on MR-Linac treatments $(95 \% \mathrm{Cl} 2.1-7.1 \mathrm{~Gy})$ compared with CBCT-Linac $(\mathrm{p}=$ $0.0027)$. There was a $1.4 \mathrm{~Gy}$ mean difference between MR-Linac Monaco modelled skin dose and in vivo OSLD skin dose. In contrast, there was a 4.0 Gy mean difference between CBCT-Linac Pinnacle modelled Loading [MathJax]/jax/output/CommonHTML/jax.js 
more accurately (Spearman's correlation $\rho=0.9500, p<0.0001$ ). By comparison, there was a weaker association between CBCT-Linac Pinnacle modelled skin dose and in vivo OSLD skin dose (Spearman's correlation $\rho=0.8000, p<0.0096$ ).

\section{Discussion}

We demonstrate that Monaco is able to accurately generate safe MR-Linac radiotherapy treatment plans for glioma patients that achieve planning objectives. We observed that for coplanar beam arrangements, MR-Linac treatments have lower homogeneity, but higher dose conformity and equivalent dose falloff outside of the target, when compared with CBCT-Linac. Conventional CBCT-Linac with non-coplanar beams can potentially achieve better dose falloff than MR-Linac, but only coplanar beam arrangements were used to standardize comparisons. MR-Linac treatment plans had more heterogenous dose distributions, which is consistent with the observed small but statistically significant increase in PTV $\mathrm{D} 50 \%, \mathrm{D} 5 \%$, and $\mathrm{D} 2 \%$. This is also consistent with previous reports showing higher heterogeneity and higher median V100\% for MR-Linac plans compared with CBCT-Linac [24]. Similarly, a very small but statistically significant increase in Brainstem D0.1cc, each Globe D0.03cc, and each Lens D0.03cc was observed in MR-Linac plans. Over the course of a patient's entire radiotherapy regimen, the absolute summed dose difference was $<1$ Gy for PTV parameters, $<1$ Gy for brainstem, approximately $3 \mathrm{~Gy}$ for each globe, and approximately $2 \mathrm{~Gy}$ for each lens. Since MR-Linac treatment plans are adapted to position every fraction [25], the exact location of these minimally higher dose regions varies geospatially every fraction, which may negate their effects when accumulated over the treatment course. Nonetheless, all MR-Linac treatments achieved standard planning objectives and dose constraints, and it is unlikely that these small differences translate into clinically relevant outcomes.

We also quantitatively characterized the impact of the MR-Linac's magnetic field on delivered dose to skin and tissue surrounding air cavities. Compared to CBCT-Linac, we observed that MR-Linac treatments showed 1.52 Gy higher Dmean $(p<0.0001)$, and 1.23 Gy higher D2cc $(p=0.0007)$ for tissues surrounding air cavities. Skin D2cc was not statistically different $(p>0.05)$, skin Dmean was 1.10 Gy higher $(p<$ $0.0001)$, and skin V20Gy was $19.04 \mathrm{~cm} 3$ higher $(p=0.0001)$ with MR-Linac treatment. This is consistent with recent preliminary studies investigating the effect of the MR-Linac's magnetic field on radiotherapy treatment [12-17]. Tseng et al. used Monaco to retrospectively generate MR-Linac plans with 9 coplanar non-opposing IMRT beams on 24 patients with intact single brain metastases, and found MR-Linac had 0.08 Gy higher Dmean and 0.6 Gy higher D2cc for skin, and 0.07 Gy higher Dmean and 0.3 Gy higher D2cc for tissues around air cavities [13]. Schrenk et al. used an open-source Monte Carlo-based TPS to retrospectively generate plans in the presence of a magnetic field with $\geq 7$ coplanar non-opposing 3DCRT and IMRT beams on 4 patients with non-small cell lung cancer, and found that the presence of the perpendicular magnetic field increased mean dose to tissues surrounding the lung air cavity by $0.5 \mathrm{~Gy}$ (18.5\%) [14]. Nachbar et al. used Monaco to prospectively generate a MR-Linac plan with 7 coplanar nonopposing IMRT beams on 1 breast cancer patient, and found MR-Linac had 0.3 Gy higher D2cc and 15.3\% higher V35Gy for skin tissue [15]. Xia et al. used Monaco to retrospectively generate MR-Linac 
cancer, and found MR-Linac had 1.30-1.81 Gy higher Dmean and 1.68-5.43 Gy higher Dmax for skin, and no difference in dose to tissues surrounding air cavities except for higher Dmax to larynx and trachea [16]. Boldrini et al. used MRIdian TPS to retrospectively generate MR-Linac plans with 7 coplanar nonopposing equally-spaced IMRT beams on 10 patients with locally advanced rectal cancer, and found MRLinac had higher skin dose and higher PTV V105\% (14.8\%) compared with CBCT-Linac VMAT with two full coplanar arcs (5.0\%) and CBCT-Linac IMRT using 5 coplanar beams (7.3\%) [17]. Taken together, these studies demonstrated that MR-Linac plans have small increases in dose to skin and tissues surrounding air cavities, and are consistent with our findings. However, the present study is unique in that the selected patient population represents a large cohort of glioma patients who received at least one fraction on both MR-Linac and CBCT-Linac based on clinically approved radiotherapy plans. Second, the plans were prospectively generated prior to treatment and delivered on both MR-Linac and CBCT-Linac, in contrast to previous studies of simulated plans that were retrospectively generated for dosimetric comparison. Lastly and importantly, we performed in vivo measurements to correlate the skin dose calculated on Monaco and Pinnacle, with measured patient skin dose via OSLD on MR-Linac and CBCT-Linac.

A potential limitation of our study is the variability in dose fractionations used. However, plan evaluation was performed with pairwise comparisons between MR-Linac and CBCT-Linac treatments for each patient and are independent of absolute values. Second, there may be potential uncertainty in OSLD measurements caused by placement, air gaps, and surface effects. To mitigate this, a single OSLD measurement was obtained from each patient's MR-Linac and CBCT-Linac treatments using standardized technique [12], although we acknowledge that reproducibility could be assessed by performing additional measurements. Third, since there are differences in how each TPS models patient surface, calculates surface dosimetry, and uses voxel sizes for TPS dose evaluation, we recognize the difficulty in quantifying the magnitude of these effects and their contribution to the dose differences observed. Finally, each patient's clinically delivered treatment plan was analyzed on the latest version of clinical Monaco TPS, and we acknowledge that future iterations of clinical Monaco may have even better dose optimization, dose engines, and dose modelling.

Potential strategies to mitigate skin dose in MR-Linac treatment include increasing the number of beam angles [26-28], using opposing beam configurations [29], using VMAT [30], using partial arcs [31], using smaller margins on target volumes [32], and specifically using a skin objective during planning optimization to minimize skin dose. Kim et al. investigated the effects of different beam arrangements on skin dosimetry for partial breast radiotherapy using MR-Linac, and demonstrated an 11-18\% increase in skin D1cc and an 146-149\% increase in skin V30 with the addition of the magnetic field. However, increasing the number of beam angles, and going from IMRT to VMAT reduced the skin dose in the presence of the magnetic field [27]. Bainbridge et al. investigated the effect of a PTV margin reduction from $7 \mathrm{~mm}$ to $3 \mathrm{~mm}$ on skin dosimetry for lung cancer radiotherapy using MR-Linac. They demonstrated that MR-Linac plans with $7 \mathrm{~mm}$ margins had 0.4 Gy higher Dmean skin dose and 1.7 Gy higher D2cc skin dose compared to CBCT-Linac plans with $7 \mathrm{~mm}$ margins. However, MR-Linac plans with a reduced margin $(3 \mathrm{~mm})$ alleviated this issue, while also decreasing other OAR metrics, and allowed isotoxic dose 
regimens and demonstrated survival outcomes similar to that of historical control [33]. Therefore, MRIguided treatment of gliomas with a reduced CTV margin and an adaptive framework not only potentially mitigates skin dose effects on the MR-Linac, but may allow for opportunities to decrease toxicities and incorporate boost strategies based on functional imaging. Finally, since this study demonstrates that Monaco's Monte Carlo dose algorithm can accurately model the near-surface dose, using the TPS IMRT optimizer with skin as an avoidance structure can reliably decrease skin dose. Future work to incorporate skin dose mitigation strategies into planning processes are being developed. Further studies investigating favorable clinical scenarios where higher dose is required to skin and superficial targets are warranted as well.

\section{Conclusion}

This is the first prospective dosimetric comparison of glioma patients clinically treated with at least one fraction on both MR-Linac and CBCT-Linac, combined with in vivo correlation to delivered dose on each platform. The dosimetric impact of the MR-Linac's magnetic field was minimal for target volumes and standard OARs. However, higher doses to tissues at skin surface and surrounding air cavities were observed for clinically delivered MR-Linac treatments. In vivo correlation of delivered skin dose was more accurately predicted by Monaco. Future MR-Linac planning processes are being designed to account for skin dosimetry and treatment delivery.

\section{Declarations}

\section{Funding:}

The authors did not receive financial support from any organization for the submitted work.

\section{Conflicts of Interest:}

MR is a co-inventor/owns intellectual property specific to the image-guidance system

on the Gamma Knife Icon outside the submitted work.

HS has received travel accommodations/expenses and honoraria from Elekta AB outside the submitted work.

SM has provided research support to Novartis AG and has received honoraria from Novartis AG and Ipsen and travel accommodations/expenses from Elekta outside the submitted work.

$B K$ has received previous grant funding from Elekta $A B$ outside of the submitted work and has also received travel accommodations/expenses from Elekta $A B$ outside of this work.

AS is an advisor/consultant for AbbVie, Merck, Roche, Varian, Elekta AB, BrainLAB, and VieCure; is a hnard momhor af tho Intornatinnal ctorontastic Radiosurgery Society; is cochair of the AO Spine Loading [MathJax]/jax/output/CommonHTML/jax.js 
Knowledge Forum Tumor; has conducted educational seminars for Elekta AB, Accuray Inc, Varian, BrainLAB, and Medtronic Kyphon; has received research grants from Elekta $A B$ and travel accommodations/expenses from Elekta $A B$, Varian, and BrainLAB; and is a member of the Elekta MRLinac Research Consortium, Elekta Spine, Oligometastases, and Linac-based SRS Consortia.

CLT has received travel accommodations/expenses and honoraria from Elekta $A B$ and belongs to the Elekta MR-Linac Research Consortium.

The remaining authors reported no disclosures or conflicts of interest.

\section{Availability of Data and Material:}

Data will be made available on request to the corresponding author following institutional ethics committee protocols.

\section{Author Contributions:}

Conceptualization: MW, AK, MR, AS, CLT

Methodology: All authors

Formal analysis and investigation: All authors

Writing - original draft preparation: MW, AS, CLT

Writing - review and editing: All authors

Project administration and supervision: AS, CLT

All the authors are in agreement and accountable for all the aspects of the work.

Clinicaltrials.gov: NCT04075305

\section{References}

1. Stewart J, Sahgal A, Lee Y, Soliman H, Tseng CL, Detsky J, Husain Z, Ho L, Das S, Maralani PJ, Lipsman N, Stanisz G, Perry J, Chen H, Atenafu EG, Campbell M, Lau AZ, Ruschin M, Myrehaug S. Quantitating Interfraction Target Dynamics During Concurrent Chemoradiation for Glioblastoma: A Prospective Serial Imaging Study. Int J Radiat Oncol Biol Phys. 2021 Mar 1;109(3):736-746. doi: 10.1016/j.jijrobp.2020.10.002. Epub 2020 Oct 14. PMID: 33068687

2. Maralani PJ, Myrehaug S, Mehrabian H, Chan AKM, Wintermark M, Heyn C, Conklin J, Ellingson BM, Rahimi S, Lau AZ, Tseng CL, Soliman H, Detsky J, Daghighi S, Keith J, Munoz DG, Das S, Atenafu EG, Lipsman N, Perry J, Stanisz G, Sahgal A (2021 Mar) Intravoxel incoherent motion (IVIM) modeling of diffusion MRI durina chemoradiation nredicts therapeutic response in IDH wildtype glioblastoma. 
Radiother Oncol 156:258-265 doi: 10.1016/j.radonc.2020.12.037. Epub 2021 Jan 5. PMID: 33418005; PMCID: PMC8186561

3. Raaymakers BW, Raaijmakers AJ, Kotte AN, Jette D, Lagendijk JJ (2004) Integrating a MRI scanner with a $6 \mathrm{MV}$ radiotherapy accelerator: dose deposition in a transverse magnetic field. Phys Med Biol. Sep 7;49(17):4109-18. doi: 10.1088/0031-9155/49/17/019. PMID: 15470926

4. Chen X, Prior P, Chen GP, Schultz CJ, Li XA. Technical Note: Dose effects of $1.5 \mathrm{~T}$ transverse magnetic field on tissue interfaces in MRI-guided radiotherapy. Med Phys. 2016 Aug;43(8):4797. doi:

10.1118/1.4959534. PMID: 27487897

5. Hackett SL, van Asselen B, Wolthaus JWH, Bluemink JJ, Ishakoglu K, Kok J, Lagendijk JJW, Raaymakers BW (2018) Spiraling contaminant electrons increase doses to surfaces outside the photon beam of an MRI-linac with a perpendicular magnetic field. Phys Med Biol. May 1;63(9):095001. doi: 10.1088/1361-6560/aaba8f. PMID: 29595150

6. Huang CY, Yang B, Lam WW, Tang KK, Li TC, Law WK, Cheung KY, Yu SK (2021) Effects on skin dose from unwanted air gaps under bolus in an MR-guided linear accelerator (MR-linac) system. Phys Med Biol. Mar 12;66(6):065021. doi: 10.1088/1361-6560/abe837. PMID: 33607641

7. Ahmad SB, Sarfehnia A, Kim A, Wronski M, Sahgal A, Keller BM (2016) Backscatter dose effects for high atomic number materials being irradiated in the presence of a magnetic field: A Monte Carlo study for the MRI linac. Med Phys 43:4665-4673

8. Ahmad SB, Sarfehnia A, Paudel MR, Kim A, Hissoiny S, Sahgal A, Keller B (2016 Feb;43(2):894-907) Evaluation of a commercial MRI Linac based Monte Carlo dose calculation algorithm with GEANT4. Med Phys. doi: 10.1118/1.4939808. PMID: 26843250

9. Paudel MR, Kim A, Sarfehnia A, Ahmad SB, Beachey DJ, Sahgal A, Keller BM. Experimental evaluation of a GPU-based Monte Carlo dose calculation algorithm in the Monaco treatment planning system. J Appl Clin Med Phys. 2016 Nov 8;17(6):230-241. doi: 10.1120/jacmp.v17i6.6455. PMID: 27929496; PMCID: PMC5690498

10. Raaijmakers AJ, Hårdemark B, Raaymakers BW, Raaijmakers CP, Lagendijk JJ. Dose optimization for the MRI-accelerator: IMRT in the presence of a magnetic field. Phys Med Biol. 2007 Dec 7;52(23):7045-54. doi: 10.1088/0031-9155/52/23/018. Epub 2007 Nov 15. PMID: 18029992

11. Raaijmakers AJ, Raaymakers BW, Lagendijk JJ. Integrating a MRI scanner with a 6 MV radiotherapy accelerator: dose increase at tissue-air interfaces in a lateral magnetic field due to returning electrons. Phys Med Biol. 2005 Apr 7;50(7):1363-76. doi: 10.1088/0031-9155/50/7/002. Epub 2005 Mar 16. PMID: 15798329

12. Kim A, Lim-Reinders S, Ahmad SB, Sahgal A, Keller BM. Surface and near-surface dose measurements at beam entry and exit in a 1.5 T MR-Linac using optically stimulated luminescence dosimeters. Phys Med Biol. 2020 Feb 12;65(4):045012. doi: 10.1088/1361-6560/ab64b6. PMID: 31860896

13. Tseng CL, Eppinga W, Seravalli E, Hackett S, Brand E, Ruschin M, Lee YK, Atenafu EG, Sahgal A (2017 Loading [MathJax]/jax/output/CommonHTML/jax.js 
brain metastases: The impact of the magnetic field. Radiother Oncol 125(2):273-279 doi: 10.1016/j.radonc.2017.09.036. Epub 2017 Oct 24. PMID: 29079310

14. Schrenk O, Spindeldreier CK, Burigo LN, Hoerner-Rieber J, Pfaffenberger A (2017 Dec) Effects of magnetic field orientation and strength on the treatment planning of nonsmall cell lung cancer. Med Phys 44(12):6621-6631. doi:10.1002/mp.12631. Epub 2017 Nov 20. PMID: 29064573

15. Nachbar M, Mönnich D, Boeke S, Gani C, Weidner N, Heinrich V, Lo Russo M, Livi L, Winter J, Tsitsekidis S, Dohm O, Thorwarth D, Zips D, De-Colle C. Partial breast irradiation with the 1.5 T MRLinac: First patient treatment and analysis of electron return and stream effects. Radiother Oncol. 2020 Apr;145:30-35. doi: 10.1016/j.radonc.2019.11.025. Epub 2019 Dec 23. PMID: 31874347

16. Xia W, Zhang K, Li M, Tian Y, Men K, Wang J, Yi J, Li Y, Dai J. Impact of Magnetic Field on Dose Distribution in MR-Guided Radiotherapy of Head and Neck Cancer. Front Oncol. 2020 Sep 8;10:1739. doi: 10.3389/fonc.2020.01739. Erratum in: Front Oncol. 2020 Nov 13;10:604231. PMID: 33014859; PMCID: PMC7506127

17. Boldrini L, Placidi E, Dinapoli N, Azario L, Cellini F, Massaccesi M, Chiesa S, Gambacorta MA, Mattiucci GC, Piccari D, Teodoli S, De Spirito M, Valentini V. Hybrid Tri-Co-60 MRI radiotherapy for locally advanced rectal cancer: An in silico evaluation. Tech Innov Patient Support Radiat Oncol. 2018 Mar 31;6:5-10. doi: 10.1016/j.tipsro.2018.02.002. PMID: 32095572; PMCID: PMC7033778

18. de Mol van Otterloo SR, Christodouleas JP, Blezer ELA, Akhiat H, Brown K, Choudhury A, Eggert D, Erickson BA, Faivre-Finn C, Fuller CD, Goldwein J, Hafeez S, Hall E, Harrington KJ, van der Heide UA, Huddart RA, Intven MPW, Kirby AM, Lalondrelle S, McCann C, Minsky BD, Mook S, Nowee ME, Oelfke U, Orrling K, Sahgal A, Sarmiento JG, Schultz CJ, Tersteeg RJHA, Tijssen RHN, Tree AC, van Triest B, Hall WA, Verkooijen HM (2020 Sep) The MOMENTUM Study: An International Registry for the Evidence-Based Introduction of MR-Guided Adaptive Therapy. Front Oncol 7:10:1328. doi:10.3389/fonc.2020.01328. PMID: 33014774; PMCID: PMC7505056

19. Stupp R, Mason WP, van den Bent MJ, Weller M, Fisher B, Taphoorn MJ, Belanger K, Brandes AA, Marosi C, Bogdahn U, Curschmann J, Janzer RC, Ludwin SK, Gorlia T, Allgeier A, Lacombe D, Cairncross JG, Eisenhauer E, Mirimanoff RO; European Organisation for Research and Treatment of Cancer Brain Tumor and Radiotherapy Groups; National Cancer Institute of Canada Clinical Trials Group. Radiotherapy plus concomitant and adjuvant temozolomide for glioblastoma. N Engl J Med (2005) Mar 10;352(10):987 - 96. doi: 10.1056/NEJMoa043330. PMID: 15758009

20. Perry JR, Laperriere N, O'Callaghan CJ, Brandes AA, Menten J, Phillips C, Fay M, Nishikawa R, Cairncross JG, Roa W, Osoba D, Rossiter JP, Sahgal A, Hirte H, Laigle-Donadey F, Franceschi E, Chinot O, Golfinopoulos V, Fariselli L, Wick A, Feuvret L, Back M, Tills M, Winch C, Baumert BG, Wick W, Ding K, Mason WP; Trial Investigators. Short-Course Radiation plus Temozolomide in Elderly Patients with Glioblastoma. N Engl J Med (2017) Mar 16;376(11):1027-1037. doi: 10.1056/NEJMoa1611977. PMID: 28296618

21. Bol GH, Kotte AN, van der Heide UA, Lagendijk JJ (2009 Nov) Simultaneous multi-modality ROI delineation in clinical practice. Comput Methods Programs Biomed 96(2):133-140.

Loading [MathJax]/jax/output/CommonHTML/jax.js 2009 May 13. PMID: 19443076

Page 16/19 
22. Tseng CL, Stewart J, Whitfield G, Verhoeff JJC, Bovi J, Soliman H, Chung C, Myrehaug S, Campbell M, Atenafu EG, Heyn C, Das S, Perry J, Ruschin M, Sahgal A (2020 Sep) Glioma consensus contouring recommendations from a MR-Linac International Consortium Research Group and evaluation of a CT-MRI and MRI-only workflow. J Neurooncol 149(2):305-314. doi:10.1007/s11060-020-03605-6. Epub 2020 Aug 29. PMID: 32860571; PMCID: PMC7541359

23. Hissoiny S, Ozell B, Bouchard H, Després P (2011 Feb;38(2):754 - 64) GPUMCD: A new GPU-oriented Monte Carlo dose calculation platform. Med Phys. doi: 10.1118/1.3539725. PMID: 21452713

24. Winkel D, Bol GH, Werensteijn-Honingh AM, Intven MPW, Eppinga WSC, Hes J, Snoeren LMW, Sikkes GG, Gadellaa-van Hooijdonk CGM, Raaymakers BW, Jürgenliemk-Schulz IM, Kroon PS (2020 May) Target coverage and dose criteria based evaluation of the first clinical 1.5T MR-linac SBRT treatments of lymph node oligometastases compared with conventional CBCT-linac treatment. Radiother Oncol 146:118-125 doi: 10.1016/j.radonc.2020.02.011. Epub 2020 Mar 6. PMID: 32146257

25. Ruschin M, Sahgal A, Tseng CL, Sonier M, Keller B, Lee Y. Dosimetric Impact of Using a Virtual Couch Shift for Online Correction of Setup Errors for Brain Patients on an Integrated High-Field Magnetic Resonance Imaging Linear Accelerator. Int J Radiat Oncol Biol Phys. 2017 Jul 1;98(3):699-708. doi: 10.1016/j.jijrobp.2017.03.004. Epub 2017 Mar 10. PMID: 28581412

26. Raaijmakers AJ, Raaymakers BW, van der Meer S, Lagendijk JJ (2007) Integrating a MRI scanner with a $6 \mathrm{MV}$ radiotherapy accelerator: impact of the surface orientation on the entrance and exit dose due to the transverse magnetic field. Phys Med Biol. Feb 21;52(4):929 - 39. doi: 10.1088/00319155/52/4/005. Epub 2007 Jan 22. PMID: 17264362

27. Kim A, Lim-Reinders S, McCann C, Ahmad SB, Sahgal A, Lee J, Keller BM (2017 Nov) Magnetic field dose effects on different radiation beam geometries for hypofractionated partial breast irradiation. $J$ Appl Clin Med Phys 18(6):62-70. doi:10.1002/acm2.12182. Epub 2017 Sep 13. PMID: 28901729; PMCID: PMC5689934

28. Lim-Reinders S, Keller BM, Sahgal A, Chugh B, Kim A. Measurement of surface dose in an MR-Linac with optically stimulated luminescence dosimeters for IMRT beam geometries. Med Phys. 2020 Jul;47(7):3133-3142. doi: 10.1002/mp.14185. Epub 2020 May 27. PMID: 32302010

29. Bol GH, Lagendijk JJ, Raaymakers BW (2015) Compensating for the impact of non-stationary spherical air cavities on IMRT dose delivery in transverse magnetic fields. Phys Med Biol. Jan 21;60(2):755 - 68. doi: 10.1088/0031-9155/60/2/755. Epub 2015 Jan 5. PMID: 25559321

30. Audet C, Poffenbarger BA, Chang P, Jackson PS, LundahI RE, Ryu SI, Ray GR. Evaluation of volumetric modulated arc therapy for cranial radiosurgery using multiple noncoplanar arcs. Med Phys. 2011 Nov;38(11):5863-72. doi: 10.1118/1.3641874. PMID: 22047350

31. Davidson MT, Masucci GL, Follwell M, Blake SJ, Xu W, Moseley DJ, Sanghera P, Wong CS, Perry J, Tsao M, Sahgal A (2012 Jun;11(3):211 - 20) Single arc volumetric modulated arc therapy for complex brain gliomas: is there an advantage as compared to intensity modulated radiotherapy or 
by adding a partial arc? Technol Cancer Res Treat. doi: 10.7785/tcrt.2012.500289. Epub 2012 Mar 1. PMID: 22376134

32. Bainbridge HE, Menten MJ, Fast MF, Nill S, Oelfke U, McDonald F (2017 Nov) Treating locally advanced lung cancer with a 1.5T MR-Linac - Effects of the magnetic field and irradiation geometry on conventionally fractionated and isotoxic dose-escalated radiotherapy. Radiother Oncol 125(2):280-285 doi: 10.1016/j.radonc.2017.09.009. Epub 2017 Oct 4. PMID: 28987747; PMCID: PMC5710994

33. Azoulay M, Chang SD, Gibbs IC, Hancock SL, Pollom EL, Harsh GR, Adler JR, Harraher C, Li G, Hayden Gephart M, Nagpal S, Thomas RP, Recht LD, Jacobs LR, Modlin LA, Wynne J, Seiger K, Fujimoto D, Usoz M, von Eyben R, Choi CYH, Soltys SG. A phase I/II trial of 5-fraction stereotactic radiosurgery with 5-mm margins with concurrent temozolomide in newly diagnosed glioblastoma: primary outcomes. Neuro Oncol. 2020 Aug 17;22(8):1182-1189. doi: 10.1093/neuonc/noaa019. PMID: 32002547; PMCID: PMC7594571

\section{Figures}
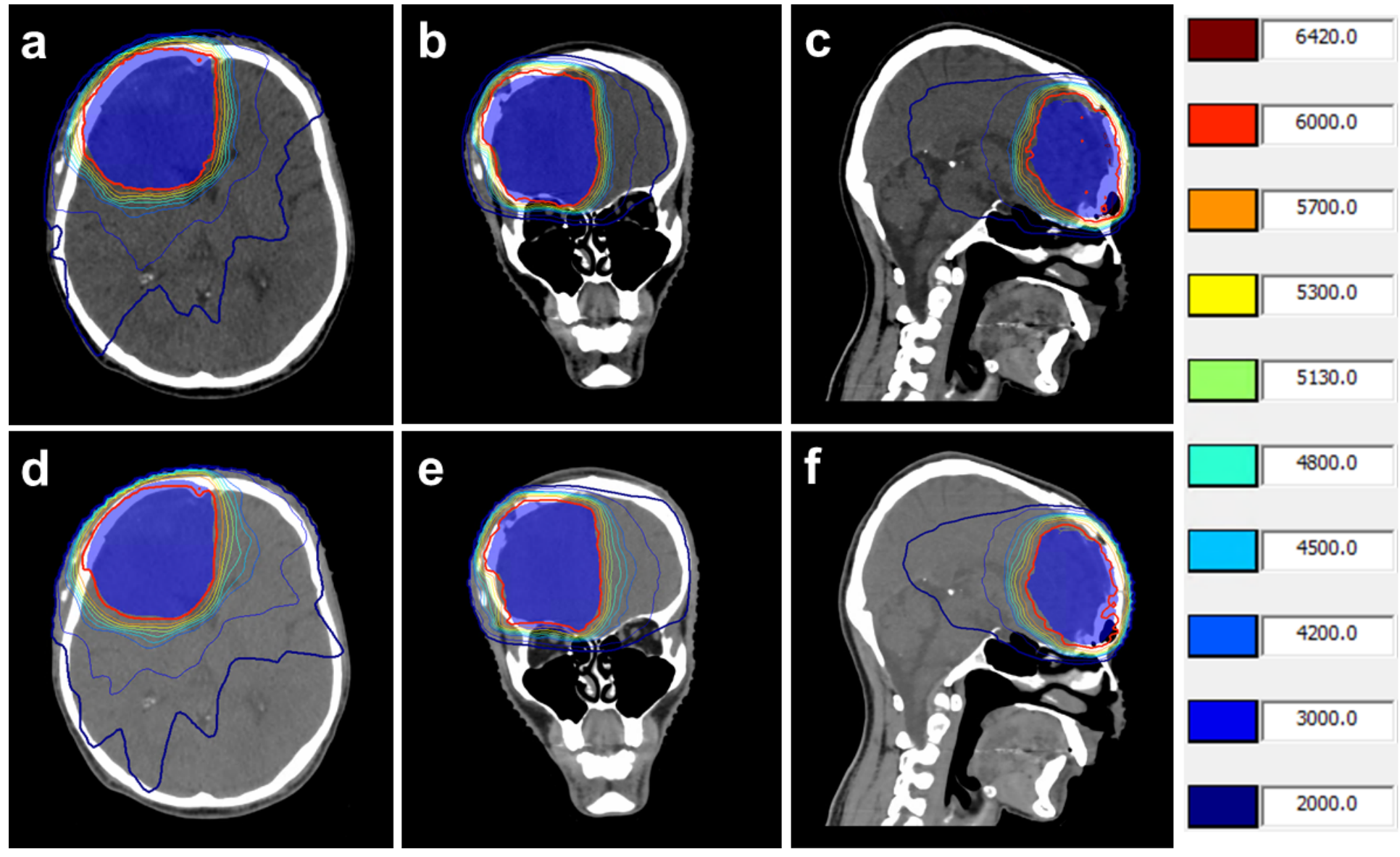

Figure 1

Clinically delivered treatment plans for a representative study patient diagnosed with glioblastoma, and Loading [MathJax]/jax/output/CommonHTML/jax.js 
distributions from Monaco. Bottom row depicts conventional Linac axial (d), coronal (e), and sagittal (f) dose distributions generated from Pinnacle. PTV is depicted in blue colorwash. Isodose lines are quantified in cGy

\section{a}

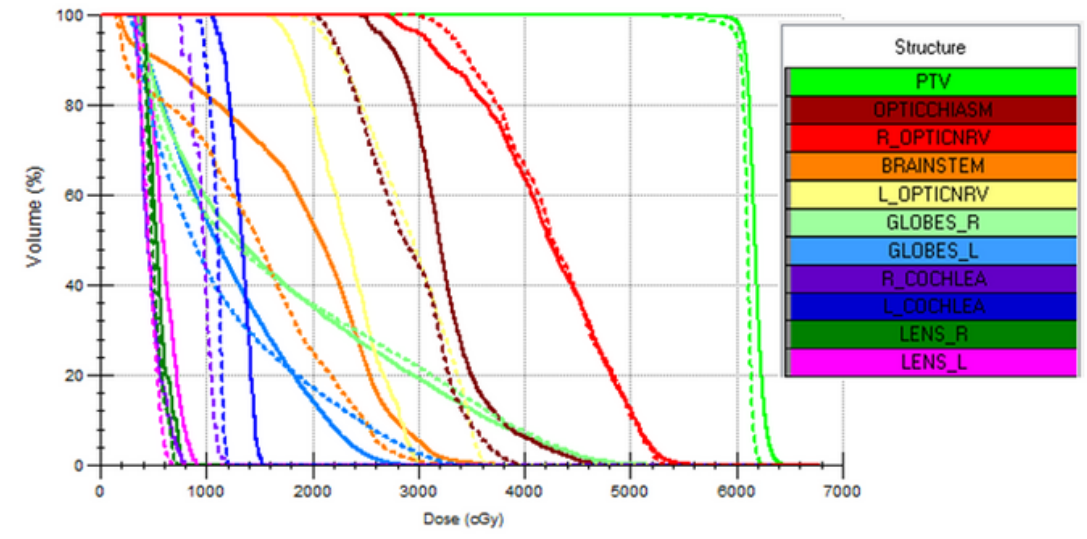

b

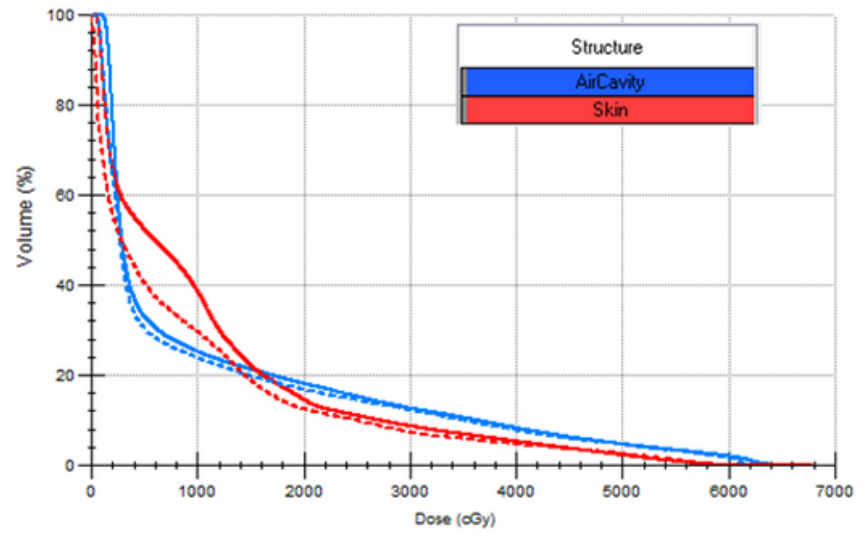

Figure 2

Dose-Volume Histograms of Planning Target Volume and Organs-at-Risk (a), as well as tissues surrounding air cavities and at skin surface (b) for a representative study patient. Delivered MR-Linac treatment (solid lines) is compared with conventional Linac treatment (dashed lines)

\section{Supplementary Files}

This is a list of supplementary files associated with this preprint. Click to download.

- SupplementalMaterial.docx 